\title{
Thoracic aortic stent grafting: Is it ready for prime time?
}

\author{
Thomas G. Gleason, MD
}

See related article on page 131.
From the Department of Surgery, Division of Cardiothoracic Surgery, Northwestern University Feinberg School of Medicine, Chicago, IL.

Received for publication Dec 13, 2005; accepted for publication Dec 13, 2005.

Address for reprints: Thomas G. Gleason, MD, Northwestern University Feinberg School of Medicine, 201 E. Huron St., Galter 10-105, 201 East Huron St, Chicago, IL 60611-3056 (E-mail: tgleason@ nmh.org).

J Thorac Cardiovasc Surg 2006;131:16-8

$0022-5223 / \$ 32.00$

Copyright () 2006 by The American Association for Thoracic Surgery

doi:10.1016/j.jtcvs.2005.09.008
$\mathrm{R}$ icco and colleagues ${ }^{1}$ are to be congratulated for their comprehensive reporting of the operative results of the French thoracic aortic stent grafting experience over the 2-year period from 1999 through 2001. This is the only report of its kind that depicts the results of an entire country's experience. It was designed by the French National Health Insurance Fund, with the data verified by third-party advisors. The report is both timely and provocative in the wake of the US Food and Drug Administration's recent approval of the use of the Gore TAG endograft for thoracic aortic aneurysms in an elective setting.

A total of 166 thoracic aortic stent grafts were placed in 29 centers, with no center performing more than 15 cases, and most centers inserting 5 or less grafts in the 2-year period. Although presumably capturing all or most thoracic aortic stent-grafting cases performed in France during the time period, the report is limited in both its scope and its analysis. The most relevant limitations are the report's retrospective nature and its inherent lack of follow-up data. Despite these flaws, the article reveals several important findings. Devices used included both industrial products (Gore [53\%], Medtronic [32\%], Stenford [4\%], and Boston Scientific [0.5\%]) and custom-made grafts designed by the implanting physicians (10\%), a profile that is representative of trends around the world. The morbidity and mortality rates by device implanted or by physician specialty (ie, surgeon, radiologist, or cardiologist) are not provided. The overall mortality and major complication rates were significant at $10 \%$ and $29 \%$, respectively (although 3 patients were not followed to 30 days). Type I or II endoleaks occurred in $16.3 \%$, but among patients who had proximal descending thoracic aortic coverage (23 patients), 26\% had a type I endoleak. Fifteen of the 23 had concomitant left subclavian artery transposition. All type I leaks required additional intervention either by means of open or endovascular techniques. The details of how and when these endoleaks were diagnosed are not provided. Two cases of proximal stent graft migration occurred, but it is not clear whether this migration occurred intraoperatively or postoperatively. None of the type II endoleaks (18 cases) were treated. Follow-up is not provided to demonstrate the natural history or prognosis of these type II leaks in this series. Type III endoleaks were not reported, although 3 of the type I leaks apparently occurred between 2 stent grafts. Paraplegia occurred in $6(3.6 \%)$ patients. Although the anatomic detail and extent of aneurysm coverage is not provided for the patients who had paraplegia, 3 had concomitant abdominal aortic aneurysm repairs. Five of these 6 patients died. Neither spinal drainage nor perioperative evoked potential monitoring was used in any case, a practice that would seem prudent in high-risk cases. Chronic type B aortic dissection was present in $20 \%$ of the cases, and the mortality rate among this cohort was $15.2 \%$. There were 17 cases of acute traumatic disruption treated with endografting, with a mortality rate of $6 \%$. There were 3 cases of postoperative aortoesophageal or aortotracheal fistulae, a complication that is less common after conventional open repair. Endografts were placed in 3 patients with known septic aneurysms, and 2 of these patients died within 30 days. Finally, the authors appropriately highlight the fact that at least 17\% of the aneurysms treated among the entire group were smaller than $50 \mathrm{~mm}$, and the mortality rate among these cases was $5 \%$.

In summary, the report by Ricco and colleagues ${ }^{1}$ provides an unbiased glance of what the results of this new technology are likely to be when applied across a wide range of pathology, experience, and expertise. Despite the many advances in the 
field of endovascular surgery, stent grafting the thoracic aorta remains at a developmental phase. Current devices are limited in their utility because of (1) their own structural flaws, (2) the limited array of graft sizes available, (3) the inherent difficulty in covering the acute angulations of the aortic arch and tortuous thoracic aortic segments, and (4) the lack of widespread expertise in endovascular techniques. There are no devices currently available that are uniformly applicable to the array of descending thoracic aortic diseases. Despite this realization, stent grafting clearly has an evolving role in thoracic aortic surgery, and results like these are encouraging.

In evaluating the current state of the art, it behooves us to scrutinize the results by comparing them with those of conventional techniques performed by major centers around the world. It is these results that should be used as the benchmark for the valuation of endovascular repair. For example, in 2004, Coselli and associates ${ }^{2}$ reported their experience after 387 consecutive open repairs of the descending thoracic aorta (emergency and elective repairs) with an overall operative mortality rate of $4.4 \%$. More than half of this series included patients in whom the entire descending thoracic aorta (extents A, B, and C) was replaced with a mortality rate of only $4 \%$. Contrarily, in this French endograft experience none of the patients had coverage of the entire thoracic aorta (extents A, B, and $\mathrm{C}$ ), yet the overall hospital mortality rate was $10 \%$. One question for the cardiothoracic community is whether we should be striving to meet outcome targets set by large-volume centers using conventional strategies before embracing a new technology that at first glance might not generate results that are improved over those of conventional strategies. There is no doubt that stent grafting can yield a seemingly good outcome with very little morbidity in certain settings, and this has been shown in other small series. Whether this technique should be used for aortic dissections, transections, small aneurysms $(<50 \mathrm{~mm})$, or septic aneurysms is still in question, with very little current data to support the practice.

Several questions remain unanswered by the current primary literature. Volume rendering and 3-dimensional reconstruction imaging modalities have not been standardized, and therefore their accuracy in the prediction of aortic diameter and shape to be used in planning stentgrafting procedures remains anecdotal, such that sizing strategies are sometimes imprecise. The optimal degree of upsizing a graft diameter relative to the target landing site's diameter is not always clear and differs depending on device manufacturer and composition of the endografts. Similarly, the minimum landing zone length is still not clear, particularly in or near the arch. We do not know how much arch angulation is too much, nor do we know whether left subclavian coverage will be durable, particularly when the landing zone is only $2 \mathrm{~cm}$ or less of graft length. Similarly, we do not know whether transposition will provide a better long-term result in cases of left subclavian coverage than simple coverage without transposition or bypass. Given the extent of angulation of the thoracic aorta, it is unclear whether modular segmented endografts are inferior or superior to singlesegment endografts. We do not know the long-term effect of the persistent radial tension applied to the aortic wall by these endografts or whether the radial tension predisposes to aortoesophageal or aortotracheal fistulae in segments of aorta that are adjacent to these structures.

Endoleaks are another major conundrum. Type I endoleaks compromise the integrity of a repair and predispose to aneurysm disruption, resulting in little benefit over expectant management. The clinical relevance and effect of type II and III endoleaks are less clear, particularly in the midterm and long term. Little is known about the safety and efficacy of treating thoracoabdominal aortic disease with stent grafts that cover mesenteric vessels. Several groups, including my own, are beginning to test these waters.

The risks of paraplegia appear to be lower in certain series, but not when comparing this current series with that of Coselli and associates. ${ }^{2}$ It seems prudent to extend and apply what we have learned about reducing the risks of paraplegia during conventional open repair to stent grafting. To date, guidelines for applying adjunctive measures have not been created. It is not clear which subset of patients will benefit from interventions like cerebrospinal fluid drainage or evoked potential monitoring to help prevent paraplegia after stent grafting.

Finally are the issues of credentialing and future investigation. Recently, a taskforce was established by the Society of Thoracic Surgeons that has suggested a credentialing protocol to help ensure patient safety. ${ }^{3}$ This concept is one based on the voluntary participation of surgeons. Important questions remain as to how the credentialing process should be organized and controlled and what the role of the professional societies, the corporations that make the devices, the hospitals or universities, and the government should be in this process. Rigorous clinical trialing must continue to protect patients and ensure that we continue to strive to achieve better results with endografts than can be achieved with open conventional procedures rather than be complacent with the current state of the art simply because the deployment of these endografts is seemingly straightforward, an enticing prospect for many physicians whose practices are in search of additional revenue sources. The answers to many of these questions remain elusive. Ricco and colleagues ${ }^{1}$ should be commended in their reporting of these French data. Despite the fact that these data are 3 years old, the report adds significantly to our understanding of 
both the potential effect and the limitations of this evolving technology.

\section{References}

1. Ricco J-B, Cau J, Marchand C, et al. Stent-graft repair for thoracic aortic disease: results of an independent nationwide study in France from 1999 to 2001. J Thorac Cardiovasc Surg. 2006;131:131-7.
2. Coselli JS, LeMaire SA, Conklin LD, Adams GJ. Left heart bypass during descending thoracic aortic aneurysm repair does not reduce the incidence of paraplegia. Ann Thorac Surg. 2004;77:1298-303.

3. Kouchoukos NT, Bavaria JE, Coselli JS, et al. Task Force on Endovascular Surgery. Guidelines for credentialing of practitioners to perform endovascular stent-grafting of the thoracic aorta. The Society of Thoracic Surgeons. Available at: www.sts.org. Accessed 2005. 